\title{
How to Recovery of Damaged Microorganisms by Supplying Several Sorts of Nutrients
}

Hideharu Shintani*

Faculty of Science and Engineering, Chuo University, Japan

\begin{abstract}
Final sterilization was conducted to health care products immediately prior to shipping and sterility assurance OF 10-6 of SAL (sterility assurance level) must be confirmed from the result of Biological indicators (BIs). The survived microorganisms after sterilization may be mostly damaged and injured microorganisms. The auxotrophic characteristics of injured microorganisms are different from those of healthy microorganisms. In that sense, the cultivation conditions and culture medium constituent of injured microorganisms must be used for confirming reproducible sterilization validation study, otherwise it may result in false negative at shipping test. This is indispensable to attain safety assurance of health care products. The evaluation of several sorts of nutrients must be studied to recover injured microorganisms and support growth reproducibly. It is also important to study for diminishing variation of culture medium performance among culture medium suppliers and that lot to lot or batch to batch variation to attain reproducible sterility assurance. These were studied by adding several sorts of agents to the culture medium inoculating injured microorganisms exposed by moist heating, dry heating, ethylene oxide gas exposure gamma-ray and electron beam irradiation. Even though sterilizing method may differ, when amino acids mixture, gucose and calcium were added to the culture medium, cultivation performance of injured spores was significantly improved and reproducible sterilization validation can be attained in success.
\end{abstract}

Keywords: Sterilization validation; Injured microorganisms; Damaged microorganisms; Sterility assurance; Auxotrophic characteristics; Biological indicator

\section{Introduction}

Before shipping healthcare products must be exposed final sterilization, if applicable, to obtain Sterility Assurance Leve1 (SAL) of $10^{-6}$ for 7 days incubation. If damaged and injured microorganisms after sterilization may survive, they may cause a false negative and grow after shipping, which cause contamination to healthcare products. In appearance it seems to be successful for sterilization validation at that time of shipping. Damaged and injured microorganisms in the culture medium requires in general more than 7 days (mostly 10 days) incubation period at relatively lower temperature, so validation study in several factors must be conducted. To avoid overlooking damaged and injured microorganisms and unsuccessful sterilization validation, the use of the appropriate (enriched) constitute of culture medium is indispensable.

For sterilization validation, in general, Soybean Casein Digest (SCD) agar (SCDA) is so often used. However, depending on supplier or difference lot of SCDA, cultivation performance significantly differed for injured spores. So, in order to attain reproducible cultivation performance and sterilization validation, we studied for what constitute of culture medium may cause differ to the injured spores in SCDA.

In this study, moist heating, dry heating, ethylene oxide gas (EOG) exposure, gamma-ray irradiation and electron beam irradiation was used to prepare damaged spores. In SCDA, several sorts of substances such as calcium, amino acids and glucose are required to add to attain reproducible SAL and successful sterility assurance [1-5].

\section{Experimental}

\section{Dry heat sterilization}

BIs for dry heating use (Bacillus atrophaeus ATCC 9372, $3 \times 10^{6}$ $\mathrm{CFU}$, colony forming unit/carrier) were purchased from Raven Co. Ltd. They were exposed at $160^{\circ} \mathrm{C}$ for 3 and $6 \mathrm{~min}$ to attain injured and damaged spores. They apply to SCD $\Lambda$ culture medium (M, D, B, ND, E, $N$ companies), incubate for 7 days and count colony. $\mathrm{M}, \mathrm{D}, \mathrm{BD}, \mathrm{E}$, and $\mathrm{N}$ companies stand Merck, Dainihon, Becton Dickinson, Eiken and Nissui companies.

Colony count can be done by using 3 sheet of BI and agitating with $30 \mathrm{ml}$ of $0.1 \%$ Tween 80 under cooling. The solution recovered and the procedure repeated three times and combined the test solutions. The test solution was step-wise 10-fold diluted to become 30-100 CFU/ plate (ISO 14161). Colony count can be done by incubating with SCDA culture medium at $35 \pm 2^{\circ} \mathrm{C}$ for 7 days.

Several sorts of agents were added to test for evaluating recovery of colony. They are calcium carbonate $\left(\mathrm{CaCO}_{3}\right)$, sodium pyruvate, D-alanine, L-alanine, Vitamin mixture, amino acids mixture, L-serine, lysozyme, casamino acid, magnesium chloride $\left(\mathrm{MgCl}_{2}\right)$, ATP, and glucose. These agents/nutrients are commercially available with pure grade.

\section{Moist heat sterilization}

BIs for moist heat sterilization use (Geobacillus stearothermophilus ATCC 7953, $1 \times 10^{6} \mathrm{CFU} /$ carrier) were purchased from Raven Co. Ltd. They were exposed at $121.1^{\circ} \mathrm{C}$ for 3 and $5 \mathrm{~min}$ to attain injured spores and apply to SCDA culture medium (M, D, BD, E and N companies), incubate and count colony at $55 \pm 2{ }^{\circ} \mathrm{C}$ for 7 days. Colony count can be done by using 3 sheet of BI and agitating with $30 \mathrm{ml}$ of $0.1 \%$ Tween

*Corresponding author: Hideharu shintani, Faculty of Science and Engineering 1-13-27, Kasuga, Japan, Tel: +81425922336; Fax: +81425922336; E-mail: shintani@mail.hinocatv.ne.jp

Received June 02, 2014; Accepted August 12, 2014; Published August 15, 2014

Citation: Shintani H (2014) How to Recovery of Damaged Microorganisms by Supplying Several Sorts of Nutrients. J Bioanal Biomed 6: 024-028. doi:10.4172/1948-593X.1000104

Copyright: (c) 2014 Shintani H. This is an open-access article distributed under the terms of the Creative Commons Attribution License, which permits unrestricted use, distribution, and reproduction in any medium, provided the original author and source are credited. 
Citation: Shintani H (2014) How to Recovery of Damaged Microorganisms by Supplying Several Sorts of Nutrients. J Bioanal Biomed 6: 024-028. doi:10.4172/1948-593X.1000104

80 under cooling. The solution recovered and the procedure repeated three times and combined the test solutions. The test solution was stepwise 10-fold diluted to become 30-100 CFU/plate (ISO 14161).

Several sorts of recovery agents were added to original SCDA culture medium. They are glucose, sodium pyruvate, calcium lactate, calcium carbonate, L-alanine, vitamin mixture, amino acids mixture, yeast extract, soluble starch, catalase and milk. These agents are commercially available with pure grade.

\section{EOG sterilization}

BIs for EOG sterilization use (B. atrophaeus ATCC 9372, 3.5×10 CFU/carrier) were purchased from Raven Co. Ltd. They were exposed at $600 \pm 30 \mathrm{mg} / \mathrm{L}, 60 \pm 10 \% \mathrm{RH}, 54 \pm 1^{\circ} \mathrm{C}$ for $3 \mathrm{~min}$ and 10 min, respectively to attain injured spores and apply to SCDA culture medium (BD, N, M companies) incubate and count colony.

Colony count can be done by using 3 sheets of $\mathrm{BI}$ and agitating with $30 \mathrm{ml}$ of $0.1 \%$ Tween 80 under cooling. The solution recovered and the procedure repeated three times and combined the test solutions. The test solution was step-wise 10-told diluted to become 30-100 CFU/ plate (ISO 14161). Colony count can be done by incubating with SCDA culture medium at $35 \pm 2{ }^{\circ} \mathrm{C}$ for 7 days.

Several sorts of recovery agents were added to the original SCDA culture medium. They are calcium carbonate $\left(\mathrm{CaCO}_{3}\right)$, L-alanine, D-alanine, amino adds mixture, vitamin mixture, sodium pyruvate and glucose. These agents are commercially available with pure grade.

\section{Gamma-ray irradiation sterilization}

BIs for gamma-ray irradiation sterilization use (Bacillus pumilus ATCC $9372,2.0 \times 10^{6} \mathrm{CFU} /$ carrier or B. megaterium spore at $1.4 \times 10^{6}$ $\mathrm{CFU} /$ carier were purchased from Raven Co. Ltd. B. pumilus and B.megaterium were exposed at $4.5 \mathrm{kGy}$. Injured spores were cultivated with SCDA from $\mathrm{N}$ company. Other procedures were identical to those of gamma-ray.

Several sorts of recovery agents were added to the original SCDA culture medium. They are calcium carbonate $\left(\mathrm{CaCO}_{3}\right)$, calcium lactate, $\mathrm{D}$-alanine, L-alanine, amino acids mixture, sodium pyruvate, soluble starch, activated carbon, vitamin mixture and glucose. These agents are commercially available with pure grade.

\section{Electron beam irradiation sterilization}

BIs for electron beam irradiation sterilization use (B. pumilus ATCC $9372,2.0 \times 10^{6} \mathrm{CFU} /$ carrier or B. megaterium spore at $1.4 \times 10^{6}$ CFU/carrier) were purchased from Raven Co. Ltd. B. pumilus and $B$. megaterium were exposed at $4.5 \mathrm{kGy}$. Injured spores were cultivated with SCDA from $\mathrm{N}$ company. Other procedures were identical to those of gamma-ray.

Several sorts of recovery agents were added to the original SCDA culture medium. They are calcium carbonate $\left(\mathrm{CaCO}_{3}\right)$, calcium lactate, D-alanine, L-alanine, amino adds mixture, sodium pyruvate, vitamin mixture and glucose. These agents are commercially available with pure grade.

\section{Result and Discussion}

\section{Dry heat sterilization}

In (Table 1), the initial population from 5 companies is presented. No significant difference of colony count can be observed when healthy

\begin{tabular}{|c|c|}
\hline Manufacturer of SCDA & Average of initial population $(\mathbf{n}=\mathbf{3})$, CFU/carrier \\
\hline D & $3.11 \times 10^{6}$ \\
\hline BD & $3.52 \times 10^{6}$ \\
\hline M & $3.43 \times 10^{6}$ \\
\hline E & $3.61 \times 10^{6}$ \\
\hline N & $3.14 \times 10^{6}$ \\
\hline
\end{tabular}

Labeled population was $3 \times 10^{6} \mathrm{CFU} /$ carrier

Table 1: Initial population of BI for dry heat sterilization and/or EOG sterilization use (B. atrosphaeus ATCC 9372).

\begin{tabular}{|c|c|c|}
\hline Manufacturer of SCDA & \multicolumn{2}{|c|}{$\begin{array}{c}\text { Average population }(\mathbf{n = 3}) \text {, CFU/carrier } \\
\text { Exposure time (min) }\end{array}$} \\
\hline & $\mathbf{3}$ min & $\mathbf{6}$ min \\
\hline D & $1.21 \times 10^{5}$ & $1.56 \times 10^{3}$ \\
\hline BD & $1.18 \times 10^{5}$ & $1.48 \times 10^{3}$ \\
\hline M & $1.20 \times 10^{5}$ & $9.50 \times 10^{2}$ \\
\hline N & $1.02 \times 10^{5}$ & $1.14 \times 10^{3}$ \\
\hline & $8.42 \times 10^{4}$ & $4.10 \times 10^{2}$ \\
\hline
\end{tabular}

Table 2a: Injured population of BI of $B$ atrophaeus ATCC 9372 exposed with dry heating at $160^{\circ} \mathrm{C}$ for $3 \mathrm{~min}$ or $6 \mathrm{~min}$, respectively.

\begin{tabular}{|c|c|c|}
\hline \multirow[t]{2}{*}{$\begin{array}{c}\text { Manufacturer of SCDA+0.5\% } \\
\text { glucose }\end{array}$} & \multicolumn{2}{|c|}{$\begin{array}{c}\text { Average population }(n=3), \text { CFU/carrier } \\
\text { Exposure time (min) }\end{array}$} \\
\hline & $3 \mathrm{~min}$ & $6 \mathrm{~min}$ \\
\hline$D$ & $1.47 \times 10^{5}$ & $1.75 \times 10^{3}$ \\
\hline $\mathrm{BD}$ & $1.42 \times 10^{5}$ & $1.82 \times 10^{3}$ \\
\hline M & $1.47 \times 10^{5}$ & $1.66 \times 10^{3}$ \\
\hline$E$ & $1.29 \times 10^{5}$ & $1.45 \times 10^{3}$ \\
\hline $\mathrm{N}$ & $1.20 \times 10^{5}$ & $8.40 \times 10^{2}$ \\
\hline
\end{tabular}

Table 2b: Injured population of BI of $B$ atrophaeus ATCC 9372 exposed with dry heating at $160^{\circ} \mathrm{C}$ for $3 \mathrm{~min}$ or $6 \mathrm{~min}$, respectively and cultured in SCDA constituent plus $0.5 \%$ glucose.

\begin{tabular}{|l|c|}
\hline Agents added & Population $(\mathbf{n}=\mathbf{3}), \mathbf{C F U} /$ carrier \\
\hline Not added $($ Control) & $2.80 \times 10^{3}$ \\
\hline $0.1 \% \mathrm{CaCO}_{3}$ & $4.61 \times 10^{3}$ \\
\hline $0.2 \%$ Calcium Lactate & $4.33 \times 10^{3}$ \\
\hline $0.1 \%$ Panvitan powder & $2.76 \times 10^{3}$ \\
\hline L-alanine at $100 \mu \mathrm{g} / \mathrm{ml}$ & $4.44 \times 10^{3}$ \\
\hline D-alanine at $100 \mu \mathrm{g} / \mathrm{ml}$ & $1.95 \times 10^{3}$ \\
\hline L-serine at $100 \mu \mathrm{g} / \mathrm{ml}$ & $2.50 \times 10^{3}$ \\
\hline $0.2 \% \mathrm{MgCl}_{2}$ & $2.86 \times 10^{3}$ \\
\hline $0.1 \%$ Amino acid mixture & $4.17 \times 10^{3}$ \\
\hline $0.5 \%$ Sodium pyruvate & $4.73 \times 10^{3}$ \\
\hline Lysozyme from egg white at $100 \mu \mathrm{g} / \mathrm{ml}$ & $2.14 \times 10^{3}$ \\
\hline ATP at $100 \mu \mathrm{g} / \mathrm{ml}$ & $2.66 \times 10^{3}$ \\
\hline
\end{tabular}

$\mathrm{BI}$ of $B$. atrophaeus ATCC 9372 was treated with dry heating at $160^{\circ} \mathrm{C}$ for $5 \mathrm{~min}$

Table 3: Recovery effect of several sorts of agents added into SCDA culture medium constituents from $\mathrm{N}$ company.

spores were used. ISO $11138-1$ approved -50 to $+30 \%$ deviations from the labeled population.

In (Table 2a), BI population exposed at $3 \mathrm{~min}$ and $6 \mathrm{~min}$ at $160^{\circ} \mathrm{C}$, respectively, was presented. In (Table 2a) 6 min exposure, the population differs significantly 6 depending on culture medium supplier (i.e. between D and N, 380\% difference). In (Table $2 \mathrm{~b}$ ), BI population after addition $0.5 \%$ glucose to SCDA presented. By adding $0.5 \%$ glucose, population significantly increased (Table $2 \mathrm{~b}$, for example $205 \%$ recovery at $6 \mathrm{~min}$ in $\mathrm{N}$ company).

In (Table 3), BI population exposed at $160^{\circ} \mathrm{C}$ for $5 \mathrm{~min}$ to $\mathrm{N}$ company SCDA culture medium, which presented minimum 
Citation: Shintani H (2014) How to Recovery of Damaged Microorganisms by Supplying Several Sorts of Nutrients. J Bioanal Biomed 6: 024-028. doi:10.4172/1948-593X.1000104

population in (Table 2a), were used incubation and added several agents to evaluate what agents may present increasing population. By adding $0.1 \% \mathrm{CaCO}_{3}, 0.2 \%$ calcium lactate, L-alanine, amino acid mixtures and sodium pyruvate indicated increased population compared with control. L-alanine presented different effect to $\mathrm{D}$-alanine and L-serine. L-alanine presented an identical effect to amino acids mixtures. L-alanine exists in nature, but $\mathrm{D}$-alanine does not, which is favorable result $[6,7]$. L-alanine may be the major contributor for increasing colony count among amino add mixtures. Alanine rasemase produce both of L-alanine and D-alanine, so synergitic effect of both $\mathrm{L}$ and $\mathrm{D}$-alanines will be much more favorable. Concerning Mg effect, our previous experiment indicated no effect to increase population contrary to calcium $[1,4]$.

\section{Moist heat sterilization}

In (Table 4), the initial population from 5 companies is presented. No significant difference could be observed, which was identical to (Table 1). In (Table 5a), BI population exposed at $3 \mathrm{~min}$ and $5 \mathrm{~min}$ at $121.1^{\circ} \mathrm{C}$, respectively, presented. In (Table $5 \mathrm{a}$ ), $3 \mathrm{~min}$ and $5 \mathrm{~min}$ exposure, population between $\mathrm{M}$ and $\mathrm{N}$ indicated $554 \%$ and $1309 \%$ difference, respectively, was observed. This variation is much over the approved range of ISO 11138-1 (-50-300\%). In (Table 5b), BI population after addition $0.5 \%$ glucose to SCDA presented. By adding $0.5 \%$ glucose, population significantly increased. Especially at $3 \mathrm{~min}$ exposure at $\mathrm{M}$ company and $5 \mathrm{~min}$ at $\mathrm{M}$ company, colony count increased $220 \%$ and $319 \%$. However, the difference of the performance of the company was observed $290 \%$ between $\mathrm{M}$ and $\mathrm{N}$ companies at 3 min and $459 \%$ between $\mathrm{M}$ and $\mathrm{N}$ companies at $5 \mathrm{~min}$ of (Table $5 \mathrm{~b}$ ). This is not negligible. $0.5 \%$ glucose addition may not be enough to recover and more than $0.5 \%$ glucose addition might be required to increase population and decrease the variation. These results indicated culture

\begin{tabular}{|c|c|}
\hline Manufacturer of SCDA & Average population $(\mathbf{n}=\mathbf{3})$, CFU/carrier \\
\hline D & $1.67 \times 10^{6}$ \\
\hline BD & $1.77 \times 10^{6}$ \\
\hline M & $1.53 \times 10^{6}$ \\
\hline E & $1.79 \times 10^{6}$ \\
\hline N & $1.61 \times 10^{6}$ \\
\hline
\end{tabular}

Labeled population was $1 \times 10^{6} \mathrm{CFU} /$ carrier

Table 4: Initial population of BI used for moist heating (G. stearothermophilus ATCC 7953).

\begin{tabular}{|c|c|c|}
\hline Manufacturer of SCDA & \multicolumn{2}{|c|}{$\begin{array}{c}\text { Average population }(\mathbf{n}=3) \text {, CFU/carrier } \\
\text { Exposure time } \mathbf{( m i n )}\end{array}$} \\
\hline & $\mathbf{3} \mathbf{~ m i n}$ & $\mathbf{5}$ min \\
\hline D & $1.89 \times 10^{4}$ & $2.37 \times 10^{3}$ \\
\hline $\mathrm{BD}$ & $1.66 \times 10^{4}$ & $1.75 \times 10^{3}$ \\
\hline $\mathrm{M}$ & $5.40 \times 10^{3}$ & $3.92 \times 10^{2}$ \\
\hline $\mathrm{E}$ & $2.36 \times 10^{4}$ & $2.84 \times 10^{3}$ \\
\hline $\mathrm{N}$ & $2.99 \times 10^{4}$ & $5.13 \times 10^{2}$ \\
\hline
\end{tabular}

Table 5a: Injured population of BI of G. stearothermophilus ATCC 7953 exposed with moist heating at $121.1^{\circ} \mathrm{C}$ for $3 \mathrm{~min}$ or $5 \mathrm{~min}$, respectively.

\begin{tabular}{|c|c|c|}
$\begin{array}{c}\text { Manufacturer of SCDA } \\
+\mathbf{0 . 5 \%} \text { glucose }\end{array}$ & \multicolumn{2}{|c|}{$\begin{array}{c}\text { Average population } \mathbf{( n = 3 )} \text {, CFU/carrier } \\
\text { Exposure time (min) }\end{array}$} \\
\hline D & \multicolumn{2}{|c|}{$\mathbf{~ m i n}$} \\
\hline BD & $1.96 \times 10^{4}$ & $2.95 \times 10^{3}$ \\
\hline M & $1.91 \times 10^{4}$ & $2.23 \times 10^{3}$ \\
\hline E & $1.19 \times 10^{4}$ & $1.25 \times 10^{3}$ \\
\hline N & $2.60 \times 10^{4}$ & $3.72 \times 10^{3}$ \\
\hline & $3.45 \times 10^{4}$ & $5.74 \times 10^{3}$ \\
\hline
\end{tabular}

Table 5b: Injured population of BI of G. stearothermophilus ATCC 7953 exposed with moist heating at $121.1^{\circ} \mathrm{C}$ for $3 \mathrm{~min}$ or $5 \mathrm{~min}$, respectively and cultured in SCDA constituent plus $0.5 \%$ glucose.

\begin{tabular}{|l|c|}
\hline Agents added & Population $(\mathbf{n}=\mathbf{3})$, CFU/carrier \\
\hline Not added (Control) & $1.02 \times 10^{3}$ \\
\hline $0.1 \% \mathrm{CaCO}_{3}$ & $2.56 \times 10^{3}$ \\
\hline $0.2 \%$ Calcium Lactate & $1.75 \times 10^{3}$ \\
\hline $0.1 \%$ Panvutan powder & $1.64 \times 10^{3}$ \\
\hline $0.1 \%$ Amino acid mixture & $1.72 \times 10^{3}$ \\
\hline ATP at $100 \mu \mathrm{\mu g} / \mathrm{ml}$ & $1.15 \times 10^{3}$ \\
\hline $0.5 \%$ Yeast extract & $9.73 \times 10^{2}$ \\
\hline $0.5 \%$ Soluble starch & $1.34 \times 10^{3}$ \\
\hline Catalase at $150 \mu \mathrm{g} / \mathrm{ml}$ & $1.15 \times 10^{3}$ \\
\hline L-alanine at $100 \mu \mathrm{g} / \mathrm{ml}$ & $1.92 \times 10^{3}$ \\
\hline $0.5 \%$ sodium pyruvate & $1.84 \times 10^{3}$ \\
\hline $1 \%$ fresh milk & $1.16 \times 10^{3}$ \\
\hline $1 \%$ skin milk & $1.55 \times 10^{3}$ \\
\hline
\end{tabular}

BI of G. stearothermophilus ATCC 7953 was treated with moist heating at $121.1^{\circ} \mathrm{C}$ for $4 \mathrm{~min}$.

Table 6: Recovery effect of several sorts of agents added into SCDA culture medium constituents from M company.

\begin{tabular}{|c|c|c|}
\hline Manufacturer of SCDA & \multicolumn{2}{|c|}{$\begin{array}{c}\text { Average population (n=3), CFU/carrier } \\
\text { Exposure time (min) }\end{array}$} \\
\hline & $\mathbf{3}$ min & $\mathbf{1 0}$ min \\
\hline BD & $1.33 \times 10^{5}$ & $3.13 \times 10^{3}$ \\
\hline $\mathrm{N}$ & $1.03 \times 10^{5}$ & $1.95 \times 10^{3}$ \\
\hline $\mathrm{M}$ & $1.13 \times 10^{5}$ & $2.18 \times 10^{3}$ \\
\hline
\end{tabular}

Initial population was $3.5 \times 10^{6} \mathrm{CFU} /$ carrier.

Table 7: Injured population of BI of B. atrophaeus ATCC 9372 qith EOG gas exposure for $3 \mathrm{~min}$ or $10 \mathrm{~min}$, respectively.

medium with rich agents for recovery of injured spores was necessary to attain reproducible sterilization validation.

In Table $6, \mathrm{BI}$ population exposed at $121.1^{\circ} \mathrm{C}$ for $4 \mathrm{~min}$ to $\mathrm{M}$ company SCDA culture medium, which presented minimum population in (Table 5a), was used and added several agents to evaluate what agents may present increased population. By adding all agents except only yeast extract, population is increased. Behavior of panvitan (vitamin mixture) differs between (Tables 3 and 6). The reason is not sure. One speculation is the difference of sterilization mechanism. Dry heat is oxidation of air and moist heat is denaturation of protein. As calcium is effective, so calcium salt is more effective than sodium salt.

Amino acid mixture is effective to increase population and among amino acid, L-alanine may be the most effectively contributed for increase of population. For culture temperature, $47^{\circ} \mathrm{C}$ culture was 4 times greater population than $55^{\circ} \mathrm{C}$ cultivation (data not shown), indicating that cultivation at lower temperature may be more favorable than the temperature applied for healthy spores. Therefore, for damaged spores, low temperature and longtime incubation may attain more colony count result $[8,9]$.

\section{EOG sterilization}

BI as EOG sterilization, B. atrophaeus ATCC 9372, was exposed at $600 \pm 90 \mathrm{mg} / \mathrm{L}, \mathrm{RH} 60 \pm 10 \%$ and temperature at $54 \pm 1^{\circ} \mathrm{C}$ for $3 \mathrm{~min}$ and $10 \mathrm{~min}$, respectively, and the population is presented in (Table 7). From (Table 7), $3 \mathrm{~min}$ is one log reduction, indicating $\mathrm{D}$ vale is $3 \mathrm{~min}$ and 10 min exposure indicated ca $3 \log$ reduction, indicating that the survivor curve is straight line, not curved or tailing phenomenon (Figure 1). D (decimal reduction) value indicates the time or does to decrease $1 \log$ reduction of colony count.

In (Table 8), BI population exposed at 3 mill and $10 \mathrm{~min}$, 
Citation: Shintani H (2014) How to Recovery of Damaged Microorganisms by Supplying Several Sorts of Nutrients. J Bioanal Biomed 6: 024-028. doi:10.4172/1948-593X.1000104

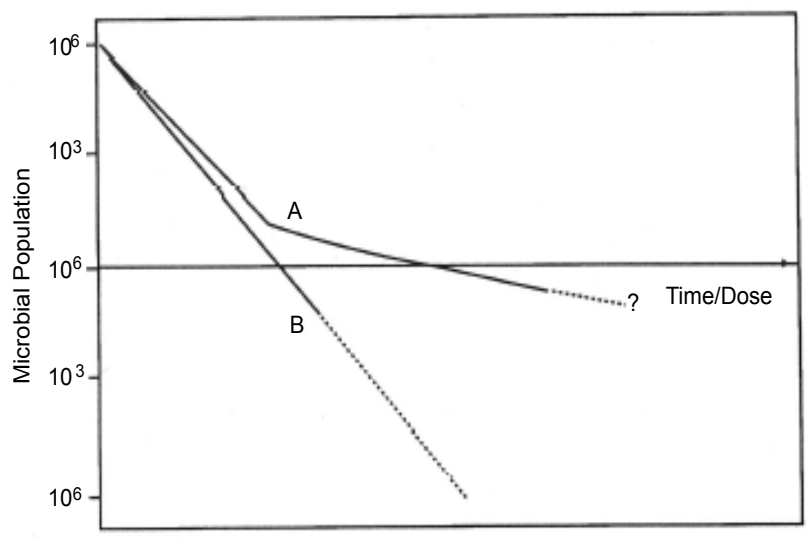

Figure 1: A: Survivor curve tailing phenomenon, B: straight line up to SLR of $10^{-6} \mathrm{SLR}$ stands spore log reduction.

\begin{tabular}{|l|c|c|}
\hline Added agents & \multicolumn{2}{|c|}{$\begin{array}{c}\text { Average population (n=3), CFU/carrier } \\
\text { Exposure time (min) }\end{array}$} \\
\hline & $\mathbf{3}$ min & $\mathbf{1 0 ~} \mathbf{~ i n}$ \\
\hline Not added (control) & $1.10 \times 10^{5}$ & $1.67 \times 10^{3}$ \\
\hline $\mathrm{CaCO}_{3}$ & $1.28 \times 10^{5}$ & $2.08 \times 10^{3}$ \\
\hline $0.2 \%$ Calcium Lactate & $1.37 \times 10^{5}$ & $2.95 \times 10^{3}$ \\
\hline $0.5 \%$ Glucose & $1.26 \times 10^{5}$ & $1.98 \times 10^{3}$ \\
\hline $0.5 \%$ Sodium pyruvate & $1.07 \times 10^{5}$ & $1.41 \times 10^{3}$ \\
\hline L-alanine at $100 \mu \mathrm{g} / \mathrm{ml}$ & $1.27 \times 10^{5}$ & $1.97 \times 10^{3}$ \\
\hline D-alanine at $100 \mu \mathrm{g} / \mathrm{ml}$ & $1.07 \times 10^{5}$ & $1.28 \times 10^{3}$ \\
\hline $0.1 \%$ Panvitan powder & $1.22 \times 10^{5}$ & $1.68 \times 10^{3}$ \\
\hline $0.1 \%$ Amono acids mixture & $1.25 \times 10^{5}$ & $1.90 \times 10^{3}$ \\
\hline
\end{tabular}

Initial population was $3.5 \times 10^{6} \mathrm{CFU} /$ carrier

Table 8: Recovery effect of several sorts of agents added into SCDA culture medium constituents from $\mathrm{N}$ company.

\begin{tabular}{|l|c|c|}
\hline Sots of microorganisms & \multicolumn{2}{|c|}{$\begin{array}{r}\text { Average population (n=3), CFU/carrier } \\
\text { Exposure condition (kGy) }\end{array}$} \\
\hline & $\mathbf{2 ~ k G y}$ & $\mathbf{5}$ kGy \\
\hline B. megaterium & $1.93 \times 10^{5}$ & $4.13 \times 10^{2}$ \\
\hline B. pumilus & $2.41 \times 10^{4}$ & $4.22 \times 10^{2}$ \\
\hline
\end{tabular}

Initial population was $1.4 \times 10^{6} \mathrm{CFU} /$ carrier

Table 9: Injured population of $B$. megaterium or $B$. pumilus exposed with gammaray at 2 or $5 \mathrm{kGy}$, respectively, cultivated in SCDA from $\mathrm{N}$ company.

respectively, were presented. BI population of $\mathrm{N}$ company SCDA culture medium, which presented minimum population in (Table 7), were used and added several agents to evaluate what agents may present increasing population. By adding all agents except sodium pyruvate and $\mathrm{D}$-alanine, population was increased. If calcium pyruvate may be used in place of sodium pyruvate, the result may favorably differ. The result of D-alanine was identical to (Table 3).

\section{Gamma-ray irradiation sterilization}

In (Table 9), the population exposed at 2 and $5 \mathrm{kGy}$ for $B$. megaterium and $B$. pumilus was presented. SCDA culture medium from $\mathrm{N}$ company, the least population in (Table 7) was used. In $B$. megaterium, $2 \mathrm{kGy}$ can be $\mathrm{D}$ value, however at $5 \mathrm{kGy}$, more than 2.5 $10 \mathrm{~g}$ reduction can be observed, indicating curved survival curve can be observed. On the contrary, D value of B. pumilus was around 1 $\mathrm{kGy}$, therefore $5 \mathrm{kGy}$ presented around $5 \log$ reduction and therefore straight line of survivor curve can be confirmed (Figure 1A).

\begin{tabular}{|l|c|c|}
\hline Added chemicals & \multicolumn{2}{|c|}{$\begin{array}{r}\text { Average population (n=3), CFU/carrier } \\
\text { Exposure condition (kGy) }\end{array}$} \\
\hline Not added (Control) & $\mathbf{2 ~ k G y}$ & $\mathbf{5}$ kGy \\
\hline $\mathrm{CaCO}_{3}$ & $1.54 \times 10^{5}$ & $2.41 \times 10^{2}$ \\
\hline $0.2 \%$ Calcium Lactate & $1.45 \times 10^{5}$ & $2.53 \times 10^{2}$ \\
\hline $0.5 \%$ Soluble starch & $1.72 \times 10^{5}$ & $3.09 \times 10^{2}$ \\
\hline $0.15 \%$ Activated charcoal & $1.46 \times 10^{5}$ & $2.51 \times 10^{2}$ \\
\hline L-alanine at $100 \mu \mathrm{g} / \mathrm{ml}$ & $1.42 \times 10^{5}$ & $2.63 \times 10^{2}$ \\
\hline D-alanine at $100 \mu \mathrm{mg} / \mathrm{ml}$ & $1.78 \times 10^{5}$ & $3.12 \times 10^{2}$ \\
\hline $0.1 \%$ Panvitan powder & $1.80 \times 10^{5}$ & $2.93 \times 10^{2}$ \\
\hline $0.1 \%$ Amino acids mixture & $1.52 \times 10^{5}$ & $2.33 \times 10^{2}$ \\
\hline $0.5 \%$ Sodium pyruvate & $1.80 \times 10^{5}$ & $2.82 \times 10^{2}$ \\
\hline $0.5 \%$ Glucose & $1.48 \times 10^{5}$ & $2.34 \times 10^{2}$ \\
\hline
\end{tabular}

Initial population was $1.4 \times 10^{6} \mathrm{CFU} /$ carrier

Table 10: Recovery effect of several sorts of chemicals onto SCDA constituents from $\mathrm{N}$ company using $B$. megaterium.

\begin{tabular}{|l|c|}
\hline Added chemicals & $\begin{array}{c}\text { Average population }(\mathbf{n}=\mathbf{3}) \text {, CFU/carrier } \\
\text { Exposure condition (kGy) }\end{array}$ \\
\hline Not added (Control) & $\mathbf{5 ~ k G y}$ \\
\hline $\mathrm{CaCO}_{3}$ & $4.84 \times 10^{2}$ \\
\hline $0.2 \%$ Calcium Lactate & $6.12 \times 10^{2}$ \\
\hline $0.5 \%$ Soluble starch & $6.33 \times 10^{2}$ \\
\hline $0.15 \%$ Activated charcoal & $4.90 \times 10^{2}$ \\
\hline L-alanine at $100 \mu \mathrm{g} / \mathrm{ml}$ & $4.80 \times 10^{2}$ \\
\hline D-alanine at $100 \mu \mathrm{g} / \mathrm{ml}$ & $6.41 \times 10^{2}$ \\
\hline $0.1 \%$ Panvitan powder & $6.92 \times 10^{2}$ \\
\hline $0.1 \%$ Amino acids mixture & $7.15 \times 10^{2}$ \\
\hline $0.5 \%$ Sodium pyruvate & $7.22 \times 10^{2}$ \\
\hline $0.5 \%$ Glucose & $6.34 \times 10^{2}$ \\
\hline
\end{tabular}

Initial population was $2 \times 10^{6} \mathrm{CFU} /$ carrier.

Table 11: Recovery effect of several sorts of chemicals added to SCDA culture medium constituent from $\mathrm{N}$ company by using $B$. pumilus spore as $\mathrm{BI}$.

In (Table 10), BI population exposed at 2 and $5 \mathrm{kGy}$ to $B$. megaterium in $\mathrm{N}$ company medium and added several sorts of agents to evaluate what agents play present increased population. By adding all agents except vitamin mixture (panvitan) and sodium pyruvate, population was increased. In place of sodium pyruvate, use of calcium pyruvate may cause more favorable result. In (Table 11), BI population exposed at $5 \mathrm{kGy}$ to B. pumilus and added several agents were conducted to evaluate what agents may present increased population. By adding all agents except activated carbon, population was increased.

\section{Electron beam irradiation sterilization}

In (Table 12), BI population exposed at $4.5 \mathrm{kGy}$ to B. megaterium and $B$. pumilus was presented by adding into $\mathrm{N}$ company culture medium and added several agents. It was conducted what agents may present increased population. By adding all agents except $\mathrm{CaCO}_{3}$, population was increased.

\section{All sterilization procedures}

In all sterilization procedures, glucose and amino add mixture were found to be favorable recovery factors. Calcium was also major candidate, but magnesium was not. Glucose will be necessary for EMP (Embden-Meyerhof-Parnas) pathway and TCA (tri carboxylic acid) cycle to attain ATP energy and several intermediate components. Amino adds and calcium cannot successfully explain. Other agents 
Citation: Shintani H (2014) How to Recovery of Damaged Microorganisms by Supplying Several Sorts of Nutrients. J Bioanal Biomed 6: 024-028. doi:10.4172/1948-593X.1000104

\begin{tabular}{|l|c|c|}
\hline Added chemicals & \multicolumn{2}{|c|}{$\begin{array}{c}\text { Average population (n=3), CFU/carrier } \\
\mathbf{4 . 5} \text { kGy exposure }\end{array}$} \\
\hline & B. megaterium & B. pumilus \\
\hline Not added (Control) & $1.78 \times 10^{3}$ & $2.51 \times 10^{3}$ \\
\hline $0.1 \% \mathrm{CaCO}_{3}$ & $1.41 \times 10^{3}$ & $2.32 \times 10^{3}$ \\
\hline $0.2 \%$ Calcium Lactate & $2.13 \times 10^{3}$ & $2.52 \times 10^{3}$ \\
\hline $0.52 \%$ Glucose & $2.17 \times 10^{3}$ & $2.70 \times 10^{3}$ \\
\hline $0.5 \%$ Sodium pyruvate & $1.88 \times 10^{3}$ & $2.68 \times 10^{3}$ \\
\hline L-alanine & $1.86 \times 10^{3}$ & $2.61 \times 10^{3}$ \\
\hline D-alanine & $2.62 \times 10^{3}$ & $2.56 \times 10^{3}$ \\
\hline $0.1 \%$ Panvitan powder & $1.93 \times 10^{3}$ & $3.06 \times 10^{3}$ \\
\hline $0.1 \%$ Amino acids mixture & $1.95 \times 10^{3}$ & $2.95 \times 10^{3}$ \\
\hline
\end{tabular}

Initial population of $B$. megaterium and that of $B$. pumilus was $1.4 \times 10^{6} \mathrm{CFU} /$ carrier and $2.0 \times 10^{6} \mathrm{CFU} /$ carrier, respectively.

Table 12: Injured population of $B$. megaterium or B. pumilus exposed with electron beam at $4.5 \mathrm{kGy}$, which cultivated in SCDA from $\mathrm{N}$ company.

indicated favorable result, but not always to other sterilization procedure. This may be the difference of the sterilization mechanism.

\section{Conclusion}

1. When used healthy spores, no significant difference of population can be observed among medium suppliers.

2. In order to recover population and reproducible validation study, calcium, glucose and amino acid mixture addition will be favorable.

3. For the incubation of injured spores. Low temperature and long period of incubation was more favorable.

4. The difference of population depending on sterilization procedures will be the difference of sterilization mechanism. Dry heat is oxidation of air, moist heat is denaturation of protein. EOG is alkylating agent, gamma-ray and electron beam are $\mathrm{OH}$ radicals, so each sterilization procedure has different mechanism for sterilization. The difference of sterilization mechanism might cause the difference of performance of agents for recovery of injured spores.

5. More than $0.5 \%$ glucose would be more favorable.

\section{References}

1. Shintani $H$ (2006) Importance of considering injured microorganisms in sterilization validation. Biocont Sci 11: 91-106.

2. Sasakj K, Shintani H, Itoh J, Kamogawa, Kajihara Y (2000) Effect of calcium in assay medium on D value of Bacillus stearothermophilus ATCC 7953 spores. Appl Environ Microbiol 66: 5509-5513.

3. Shinlani H, Sasaki K, Kajiwara Y, Itoh J, Takahashi, et al. (2000) Validation of $D$ value by different SCD culture medium manufacturer and/or different SCD culture medium constituent. PDA J Pharm Scj Technol 54: 6-12.

4. Hurst A, Hughes A (1981) Repair of salt tolerance and recovery of lost D-alanine and magnesium following sublethal heating of Staphylococcus aureus are independent events. Can J Microbiol 27: 627-632.

5. Bender GR, Marquis RE (1985) Spore heat resistance and specific mineralization. Appl Environ Microbiol 50: 1414-1421.

6. Milligan DL, Tran SL, Strych U, Cook GM, Krause KL (2007) The alanine racemase of Mycobacterium smegmatis is essential for growth in the absence of D-alanine. J Bacteriol 189: 8381-8386.

7. Gould GW (1970) Symposium on bacterial spores: IV Germination and the problem of dormancy. J Appl Bact 33: 34-49.

8. Bathgate H, Lazzari D, Cameron H, McKay D (1993) The incubation period in sterility testing. J Parenter Sci Technol 47: 254-257.

9. Besajew C (1992) Importance of incubation time in the test for sterility. Pharm Ind 54: 539-542. 